\title{
List of Deep Learning Models
}

\author{
Amir Mosavi ${ }^{1,2 *}$, Sina Ardabili ${ }^{3}$ and Annamaria R. Varkonyi-Koczy ${ }^{4}$ \\ ${ }^{I}$ Institue of Automation, Kalman Kando Faculty of Electrical Engineering, \\ Obuda University, Budapest, Hungary \\ ${ }^{2}$ School of the Built Environment, Oxford Brookes University, Oxford OX3 \\ $O B P, U K$ \\ ${ }^{3}$ Institute of Advanced Studies Koszeg, Koszeg, Hungary \\ ${ }^{4}$ Department of Mathematics and Informatics, J. Selye University, Ko- \\ marno, Slovakia \\ *amir.mosavi@kvk.uni-obuda.hu
}

\begin{abstract}
Deep learning (DL) algorithms have recently emerged from machine learning and soft computing techniques. Since then, several deep learning (DL) algorithms have been recently introduced to scientific communities and are applied in various application domains. Today the usage of DL has become essential due to their intelligence, efficient learning, accuracy and robustness in model building. However, in the scientific literature, a comprehensive list of DL algorithms has not been introduced yet. This paper provides a list of the most popular DL algorithms, along with their applications domains.
\end{abstract}

Keywords: Deep learning, machine learning, convolutional neural networks (CNN) recurrent neural networks (RNN), denoising autoencoder (DAE), deep belief networks (DBNs), long short-term memory (LSTM), review, survey, state of the art, 


\section{Introduction}

There has been an enormous evolution in system modeling and intelligence after introducing the early models for deep learning [1-8]. Deep learning methods very fast emerged and expanded applications in various scientific and engineering domains. Health informatics, energy, urban informatics, safety, security, hydrological systems modeling, economic, bioinformatics, and computational mechanics have been among the early application domains of deep learning. State of the art surveys on the data-driven methods and machine learning algorithms, e.g., [9-26], indicates that deep learning, along with the ensemble and hybrid machine learning methods are the future of data science. Further comparative studies, e.g., [26-42], report that deep learning models and hybrid machine learning models often outperform conventional machine learning models. Figure 1 represents the rapid rise in the applications of various deep learning methods during the past five years.

Deep learning methods are fast evolving for higher performance. Literature includes adequate review papers on the progressing algorithms in particular application domains, e.g., renewable energy forecasting, cardiovascular image analysis, super-resolution imaging, radiology, 3D sensed data classification, 3D sensed data classification, multimedia analytics, sentiment classification, text detection, transportation systems, activity recognition in radar, hyperspectral, medical ultrasound analysis, image cytometry, and apache spark [43-59]. However, a simplified list of deep learning methods has not been communicated so far. Thus, there is a gap in research in introducing the deep learning methods and summarize the methods and application in a brief, yet communicative paper. Consequently, this paper aims at providing a comprehensive list of the most popular deep learning methods and their notable applications. In every section, one deep learning method is introduced and the notable applications related to 
that method are listed. The description of each deep learning method and the function of each building block is explained.

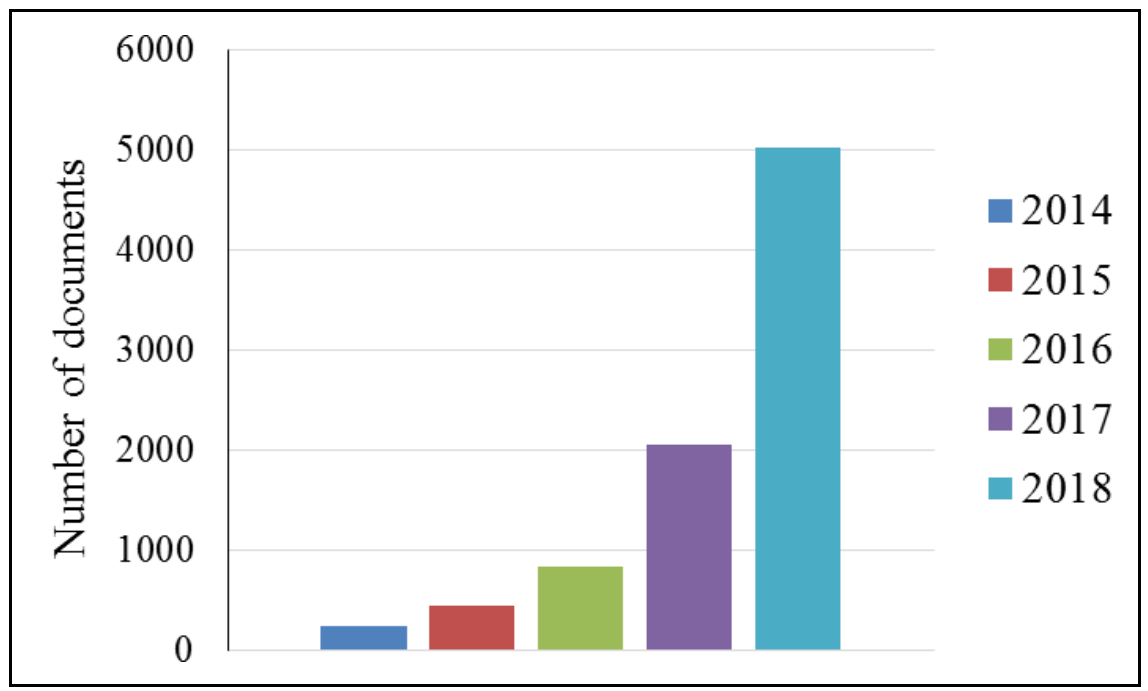

Fig. 1. The rapid increase of using DL models in various application domains (source: web of science)

\section{Deep learning methods}

Convolutional neural network (CNN) Recurrent neural network (RNN), Denoising autoencoder (DAE), deep belief networks (DBNs), Long Short-Term Memory (LSTM) are the most popular deep learning methods have been widely used. In this section, the description of each method is described along with the notable applications. 


\subsection{Convolutional neural network $(\mathrm{CNN})$}

CNN is one of the most known architectures of DL techniques. This technique is generally employed for image processing applications. CNN contains three types of layers with different convolutional, pooling, and fully connected layers (Fig. 1). In each CNN, there are two stages for the training process, the feed-forward stage, and the back-propagation stage. The most common CNN architectures are ZFNet [60], GoogLeNet [61], VGGNet [62], AlexNet [63], ResNet [64].

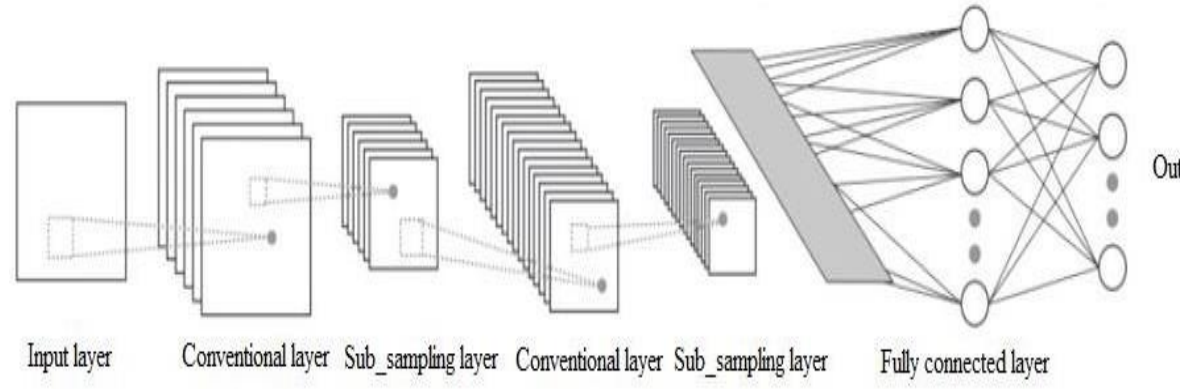

Fig. 2. CNN Architecture

Table 1. The CNN notable applications

\begin{tabular}{|l|l|l|}
\hline Reference & Application & Journal \\
\hline Kong et al. 2020[65] & $\begin{array}{l}\text { Condition monitoring of } \\
\text { wind turbines }\end{array}$ & $\begin{array}{l}\text { Renewable En- } \\
\text { ergy }\end{array}$ \\
\hline
\end{tabular}




\begin{tabular}{|l|l|l|} 
Lossau et al. 2019 [66] & $\begin{array}{l}\text { Motion estimation and } \\
\text { correction of medical im- } \\
\text { aging }\end{array}$ & $\begin{array}{l}\text { Computerized } \\
\text { Medical Imaging } \\
\text { and Graphics }\end{array}$ \\
\hline $\begin{array}{l}\text { Bhatnagar et al. 2019 } \\
\text { [67] }\end{array}$ & $\begin{array}{l}\text { Prediction of aerody- } \\
\text { namic flow }\end{array}$ & $\begin{array}{l}\text { Computational } \\
\text { Mechanics }\end{array}$ \\
\hline $\begin{array}{l}\text { Nevavuori et al. 2019 } \\
\text { [68] }\end{array}$ & Crop yield prediction & $\begin{array}{l}\text { Agriculture } \\
\text { Computers and }\end{array}$ \\
\hline Ajami et al. 2019 [69] & $\begin{array}{l}\text { Advanced image pro- } \\
\text { cessing }\end{array}$ & Remote Sensing \\
\hline
\end{tabular}

Although $\mathrm{CNN}$ is primarily known for image processing applications, the literature includes other application domains, e.g., energy, computational mechanics, electronics systems, remote sensing, etc.

\subsection{Recurrent neural networks (RNN)}

RNN is designed to recognize sequences and patterns such as speech, handwriting, text, and such applications. RNN benefits cyclic connections in the structure which employ recurrent computations to sequentially process the input data [70]. RNN is basically a standard neural network that has been extended across time by having edges which feed into the next time step instead of into the next layer in the same time step. Each of the previous inputs data are kept in a state vector in hidden units, and these state vectors is utilized to compute the outputs. Fig 2 shows the architecture of RNN. 


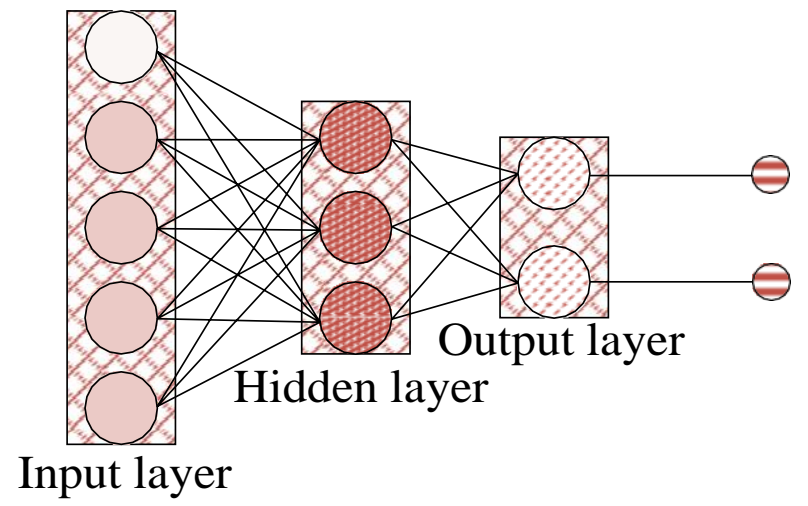

Fig. 3. RNN Architecture

Table 2. Notable RNN applications

\begin{tabular}{|l|l|l|}
\hline Reference & Application & Journal \\
\hline Zhu et al. 2019 [71] & Wind speed prediction & $\begin{array}{l}\text { Energy Conversion and } \\
\text { Management }\end{array}$ \\
\hline Pan et al. 2019 [72] & $\begin{array}{l}\text { Tropical cyclone inten- } \\
\text { sity prediction }\end{array}$ & Electronics Letters \\
\hline $\begin{array}{l}\text { Bisharad et al. 2019 } \\
\text { [73] }\end{array}$ & $\begin{array}{l}\text { Music genre recogni- } \\
\text { tion }\end{array}$ & Expert Systems \\
\hline
\end{tabular}




\begin{tabular}{|l|l|l|} 
Zhong et al. 2019 [74] & $\begin{array}{l}\text { Ship Trajectory Resto- } \\
\text { ration }\end{array}$ & Journal of Navigation \\
\hline & Stock price trends pre- & $\begin{array}{l}\text { Advanced Computer } \\
\text { Science and Applica- } \\
\text { tions }\end{array}$ \\
\hline Jarrah et al. 2019 [75] & dict & \\
\hline
\end{tabular}

RNN is relatively newer deep learning method. This is why the application domains are still young and plenty of rooms remains for research and exploration. The energy, hydrological prediction, expert systems, navigation, and economics are the current applications reported in the literature.

\subsection{Denoising AutoEncoder (DAE)}

DAE has been extended from AE as asymmetrical neural network for learning features from noisy datasets. DAE consists of three main layers, including input, encoding, and decoding layers [76]. DAE is able to be aggregated for taking high-level features. Stacked Denoising AutoEncoder (SDAE), as an unsupervised algorithm, is generated by the DEA method, which can be employed for nonlinear dimensionality reduction. This method is a type of feed-forward neural network and employs a deep architecture with multiple hidden layers and a pre-training strategy [77, 78]. Fig. 3 presents the architecture of DEA methodology. 


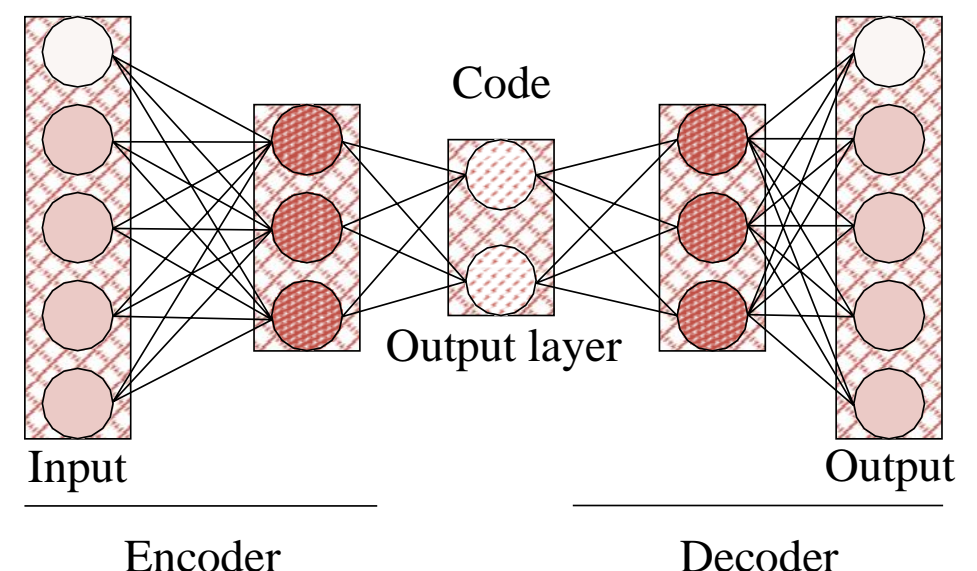

Encoder

Decoder

Fig. 4. DEA Architecture

Table 3. The notable DEA applications

\begin{tabular}{|l|l|l|}
\hline Reference & Application & Journal \\
\hline $\begin{array}{l}\text { Chen et al. 2019 } \\
{[79]}\end{array}$ & $\begin{array}{l}\text { Improving the cyber- } \\
\text { physical systems }\end{array}$ & $\begin{array}{l}\text { Journal on Wireless Com- } \\
\text { munications }\end{array}$ \\
\hline $\begin{array}{l}\text { Liu et al. 2019 } \\
{[80]}\end{array}$ & Electric load forecasting & Energies \\
\hline $\begin{array}{l}\text { Nicolai et al. } \\
2018 \text { [81] }\end{array}$ & $\begin{array}{l}\text { Laser-based scan regis- } \\
\text { tration }\end{array}$ & $\begin{array}{l}\text { IEEE Robotics and Auto- } \\
\text { mation }\end{array}$ \\
\hline
\end{tabular}




\begin{tabular}{|l|l|l|}
$\begin{array}{l}\text { Yue, et al. 2018 } \\
{[82]}\end{array}$ & Collaborative Filtering & $\begin{array}{l}\text { Computer Science and } \\
\text { Technology }\end{array}$ \\
\hline $\begin{array}{l}\text { Roy et a. 2018 } \\
{[83]}\end{array}$ & $\begin{array}{l}\text { Noisy image classifica- } \\
\text { tion }\end{array}$ & $\begin{array}{l}\text { Journal of Information } \\
\text { and Communication } \\
\text { Technology }\end{array}$ \\
\hline $\begin{array}{l}\text { Tan et al. } 2018 \\
{[84]}\end{array}$ & $\begin{array}{l}\text { Robust Speaker Verifi- } \\
\text { cation }\end{array}$ & $\begin{array}{l}\text { IEEE Transactions on Au- } \\
\text { dio Speech }\end{array}$ \\
& & \\
\hline
\end{tabular}

DEA is slowly starting to be known among researchers as an efficient DL algorithm. DEA has already been used in various application domains with promising results. The energy forecasting, cybersecurity, banking, fraud detection, image classification, and speaker verification are among the current popular applications of DEA.

\subsection{The deep belief networks (DBNs)}

DBNs are employed for high dimensional manifolds learning of data. This method contains multiple layers, including connections between the layers except for connections between units within each layer. DBNs can be considered as a hybrid multi-layered neural network, including directed and undirected connections. DBNs contains restricted Boltzmann machines (RBMs) which are trained in a greedy manner. Each RBM layer communicates with both the previous and subsequent layers $[78,85,86]$. This model is consists of a feed-forward network and several layers of restricted Boltzmann machines or RBM as 
feature extractors [87]. A hidden layer and visible layer are only two layers of an RBM [88]. Fig. 4 presents the architecture of the DBN method.

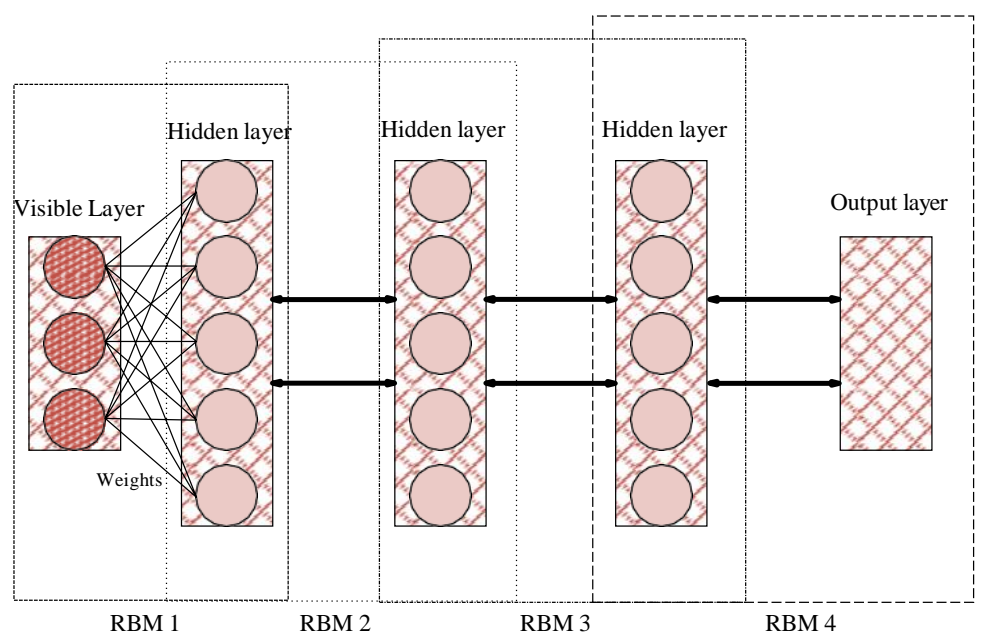

Fig. 5. DBN Architecture

Table 4. The notable DBN applications

\begin{tabular}{|l|l|l|}
\hline Reference & Application & Journal \\
\hline $\begin{array}{l}\text { Hassan et al. 2019 } \\
{[89]}\end{array}$ & $\begin{array}{l}\text { Human emotion recogni- } \\
\text { tion }\end{array}$ & Information Fusion \\
\hline $\begin{array}{l}\text { Cheng et al. 2019 } \\
{[90]}\end{array}$ & Time series prediction & IEEE Internet of Things \\
\hline
\end{tabular}




\begin{tabular}{|l|l|l|} 
Yu et al. 2019 [91] & wind speed prediction & $\begin{array}{l}\text { IEEE Transactions on } \\
\text { Electrical Engineering }\end{array}$ \\
\hline $\begin{array}{l}\text { Zheng et al. 2019 } \\
{[92]}\end{array}$ & Exchange rate forecasting & $\begin{array}{l}\text { Neural Computing and } \\
\text { Applications }\end{array}$ \\
\hline $\begin{array}{l}\text { Ahmad et al. 2019 } \\
{[93]}\end{array}$ & $\begin{array}{l}\text { Automatic Liver Segmen- } \\
\text { tation }\end{array}$ & IEEE Access \\
\hline $\begin{array}{l}\text { Ronoud et al. 2019 } \\
{[94]}\end{array}$ & Breast cancer diagnosis & Soft Computing \\
\hline
\end{tabular}

DBN is one of the most reliable deep learning methods with high accuracy and computational efficiency. Thus, the application domains have been divers, including exciting application in a wide range of engineering and scientific problems. Human emotion detection, time series prediction, renewable energy prediction, economic forecasting, and cancer diagnosis have been among the public application domains.

\subsection{Long Short-Term Memory (LSTM)}

LSTM is an RNN method which benefits feedback connections to be used as a general-purpose computer. This method can of for both sequences and patterns recognition and image processing applications. In general, LSTM contains three central units, including input, output, and forget gates. LSTM can control on deciding when to let the input enter the neuron and to remember what was computed in the previous time step. One of the main strength of the LSTM 
method is that it decides all these based on the current input itself. Fig. 6 presents the architecture of the LSTM method.

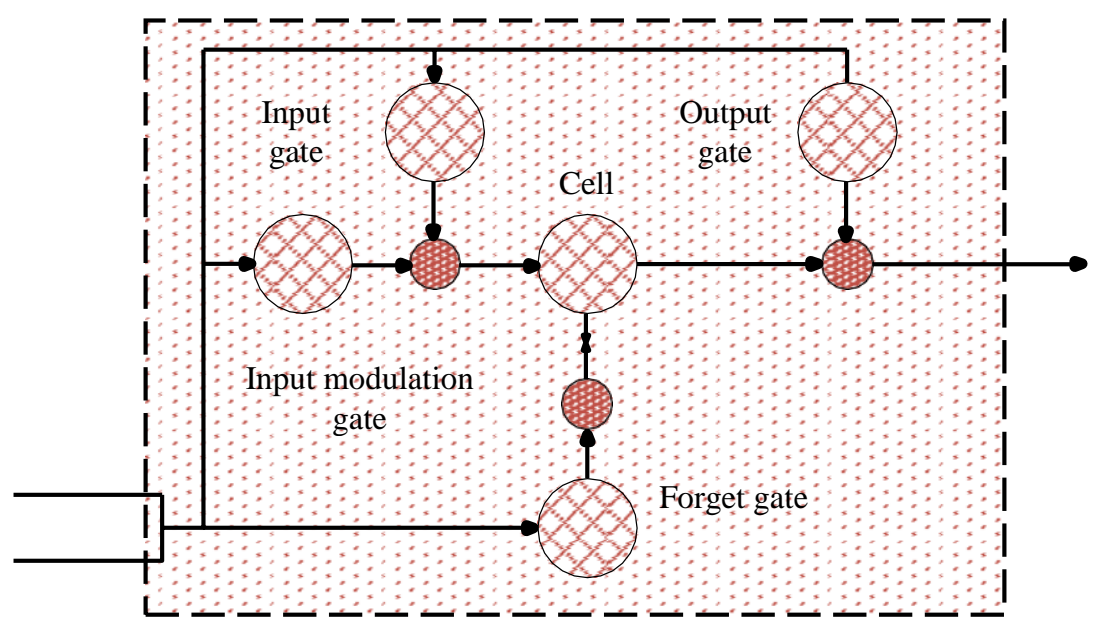

Fig. 6. LSTM Architecture

Table 5. The notable applications of LSTM

\begin{tabular}{|l|l|l|}
\hline Reference & Application & Journal \\
\hline $\begin{array}{l}\text { Ghimire et al. } \\
2019[95]\end{array}$ & $\begin{array}{l}\text { Solar radiation fore- } \\
\text { casting }\end{array}$ & Applied Energy \\
\hline Liu 2019[3] & Volatility forecasting & $\begin{array}{l}\text { Expert Systems with Ap- } \\
\text { plications }\end{array}$ \\
\hline
\end{tabular}




\begin{tabular}{|c|c|c|}
\hline $\begin{array}{l}\text { Hong et al. } 2019 \\
{[96]}\end{array}$ & $\begin{array}{l}\text { Fault prognosis of bat- } \\
\text { tery systems }\end{array}$ & Applied Energy \\
\hline $\begin{array}{l}\text { Krishan } 2019 \\
\text { [97] }\end{array}$ & Air quality prediction & $\begin{array}{l}\text { Air Quality and Atmos- } \\
\text { phere }\end{array}$ \\
\hline $\begin{array}{l}\text { Zhang et al. } 2019 \\
\text { [98] }\end{array}$ & $\begin{array}{l}\text { Structural seismic pre- } \\
\text { diction }\end{array}$ & Computers and Structures \\
\hline $\begin{array}{l}\text { Hua et al. } 2019 \\
\text { [99] }\end{array}$ & Time Series Prediction & IEEE Communications \\
\hline $\begin{array}{l}\text { Zhang et al. } 2019 \\
{[100]}\end{array}$ & $\begin{array}{l}\text { Wind turbine power } \\
\text { prediction }\end{array}$ & Applied Energy \\
\hline $\begin{array}{l}\text { Vardaan et al. } \\
2019[101]\end{array}$ & $\begin{array}{l}\text { Earthquake trend pre- } \\
\text { diction }\end{array}$ & $\begin{array}{l}\text { Electrical and Computer } \\
\text { Engineering }\end{array}$ \\
\hline
\end{tabular}

LSTM has shown great potential in environmental applications, e.g., geological modeling, hydrological prediction, air quality, and hazard modeling. Due to the generalization ability of the LSTM architecture, it can be suitable for many application domains. Energy demand and consumption, wind energy industry, and solar power modeling are the other application domains of LSTM. Further investigation is essential to explore the new deep learning methods and explore the application domains, as it has been done for machine Learning methods [102-109]. 


\section{Conclusions}

Deep learning methods are fast-evolving. Some of them have advanced to be specialized in a particular application domain. However, there is a gap in research in introducing the deep learning methods and summarize the methods and application in a single paper. Consequently, this paper aims at providing a comprehensive list of the most popular deep learning methods and provide notable applications. CNN, RNN, DAE, DBNs, LSTM methods have been identified as the most popular deep learning method. The description of each deep learning method and the function of each building block of them is explained.

\section{Acknowledgments}

This publication has been supported by the Project: "Support of research and development activities of the J. Selye University in the field of Digital Slovakia and creative industry" of the Research \& Innovation Operational Programme (ITMS code: NFP313010T504) co-funded by the European Regional Development Fund.

\section{References}

1. Diamant, A., et al., Deep learning in head \& neck cancer outcome prediction. Scientific Reports, 2019. 9(1).

2. Dong, Y., et al., Bandgap prediction by deep learning in configurationally hybridized graphene and boron nitride. npj Computational Materials, 2019. 5(1). 
3. Liu, Y., Novel volatility forecasting using deep learning-Long Short Term Memory Recurrent Neural Networks. Expert Systems with Applications, 2019. 132: p. 99-109.

4. Ludwiczak, J., et al., PiPred - a deep-learning method for prediction of $\pi$-helices in protein sequences. Scientific Reports, 2019. 9(1).

5. Matin, R., C. Hansen, and P. Mølgaard, Predicting distresses using deep learning of text segments in annual reports. Expert Systems with Applications, 2019. 132: p. 199-208.

6. Nguyen, D., et al., A feasibility study for predicting optimal radiation therapy dose distributions of prostate cancer patients from patient anatomy using deep learning. Scientific Reports, 2019. 9(1).

7. Shickel, B., et al., DeepSOFA: A Continuous Acuity Score for Critically Ill Patients using Clinically Interpretable Deep Learning. Scientific Reports, 2019. 9(1).

8. Wang, K., X. Qi, and H. Liu, A comparison of day-ahead photovoltaic power forecasting models based on deep learning neural network. Applied Energy, 2019. 251.

9. Aram, F., et al., Design and validation of a computational program for analysing mental maps: Aram mental map analyzer. Sustainability (Switzerland), 2019. 11(14).

10. Asadi, E., et al., Groundwater Quality Assessment for Drinking and Agricultural Purposes in Tabriz Aquifer, Iran. 2019.

11. Asghar, M. Z.; Subhan, F.; Imran, M.; Kundi, F.M.; Shamshirband, S.; Mosavi, A.; Csiba, P.; R. Várkonyi-Kóczy, A. Performance Evaluation 
of Supervised Machine Learning Techniques for Efficient Detection of Emotions from Online Content. Preprints 2019, 2019080019 (doi: 10.20944/preprints201908.0019.v1).

12. Bemani, A.; Baghban, A.; Shamshirband, S.; Mosavi, A.; Csiba, P.; Várkonyi-Kóczy, A.R. Applying ANN, ANFIS, and LSSVM Models for Estimation of Acid Sol-vent Solubility in Supercritical CO2. Preprints 2019, 2019060055 (doi: 10.20944/preprints201906.0055.v2).

13. Choubin, B., et al., Snow avalanche hazard prediction using machine learning methods. Journal of Hydrology, 2019. 577.

14. Choubin, B., et al., An ensemble prediction of flood susceptibility using multivariate discriminant analysis, classification and regressiontrees, and support vector machines. Science of the Total Environment, 2019. 651: p. 2087-2096.

15. Dehghani, M., et al., Prediction of hydropower generation using Grey wolf optimization adaptive neuro-fuzzy inference system. Energies, 2019. 12(2).

16. Dineva, A., et al., Review of soft computing models in design and control of rotating electrical machines. Energies, 2019. 12(6).

17. Dineva, A., et al., Multi-Label Classification for Fault Diagnosis of Rotating Electrical Machines. 2019.

18. Farzaneh-Gord, M., et al., Numerical simulation of pressure pulsation effects of a snubber in a CNG station for increasing measurement accuracy. Engineering Applications of Computational Fluid Mechanics, 2019. 13(1): p. 642-663. 
19. Ghalandari, M., et al., Investigation of submerged structures' flexibility on sloshing frequency using a boundary element method and finite element analysis. Engineering Applications of Computational Fluid Mechanics, 2019. 13(1): p. 519-528.

20. Ghalandari, M., et al., Flutter speed estimation using presented differential quadrature method formulation. Engineering Applications of Computational Fluid Mechanics, 2019. 13(1): p. 804-810.

21. Karballaeezadeh, N., et al., Prediction of remaining service life of pavement using an optimized support vector machine (case study of Semnan-Firuzkuh road). Engineering Applications of Computational Fluid Mechanics, 2019. 13(1): p. 188-198.

22. Menad, N.A., et al., Modeling temperature dependency of oil - water relative permeability in thermal enhanced oil recovery processes using group method of data handling and gene expression programming. Engineering Applications of Computational Fluid Mechanics, 2019. 13(1): p. 724-743.

23. Mohammadzadeh, S., et al., Prediction of Compression Index of FineGrained Soils Using a Gene Expression Programming Model. Infrastructures, 2019. 4(2): p. 26.

24. Mosavi, A. and M. Edalatifar, A Hybrid Neuro-Fuzzy Algorithm for Prediction of Reference Evapotranspiration, in Lecture Notes in Networks and Systems. 2019, Springer. p. 235-243.

25. Mosavi, A., A. Lopez, and A.R. Varkonyi-Koczy, Industrial applications of big data: State of the art survey, D. Luca, L. Sirghi, and C. Costin, Editors. 2018, Springer Verlag. p. 225-232. 
26. Mosavi, A., P. Ozturk, and K.W. Chau, Flood prediction using machine learning models: Literature review. Water (Switzerland), 2018. 10(11).

27. Mosavi, A. and T. Rabczuk, Learning and intelligent optimization for material design innovation, D.E. Kvasov, et al., Editors. 2017, Springer Verlag. p. 358-363.

28. Mosavi, A., T. Rabczuk, and A.R. Varkonyi-Koczy, Reviewing the novel machine learning tools for materials design, D. Luca, L. Sirghi, and C. Costin, Editors. 2018, Springer Verlag. p. 50-58.

29. Mosavi, A., et al., State of the art of machine learning models in energy systems, a systematic review. Energies, 2019. 12(7).

30. Mosavi, A., et al., Prediction of multi-inputs bubble column reactor using a novel hybrid model of computational fluid dynamics and machine learning. Engineering Applications of Computational Fluid Mechanics, 2019. 13(1): p. 482-492.

31. Mosavi, A. and A.R. Varkonyi-Koczy, Integration of machine learning and optimization for robot learning, R. Jablonski and R. Szewczyk, Editors. 2017, Springer Verlag. p. 349-355.

32. Nosratabadi, S., et al., Sustainable business models: A review. Sustainability (Switzerland), 2019. 11(6).

33. Qasem, S.N., et al., Estimating daily dew point temperature using machine learning algorithms. Water (Switzerland), 2019. 11(3). 
34. Rezakazemi, M., A. Mosavi, and S. Shirazian, ANFIS pattern for molecular membranes separation optimization. Journal of Molecular Liquids, 2019. 274: p. 470-476.

35. Riahi-Madvar, H., et al., Comparative analysis of soft computing techniques RBF, MLP, and ANFIS with MLR and MNLR for predicting grade-control scour hole geometry. Engineering Applications of Computational Fluid Mechanics, 2019. 13(1): p. 529-550.

36. Shabani, S.; Samadianfard, S.; Taghi Sattari, M.; Shamshirband, S.; Mosavi, A.; Kmet, T.; R. Várkonyi-Kóczy, A. Modeling Daily Pan Evaporation in Humid Cli-mates Using Gaussian Process Regression. Preprints 2019, 2019070351 (doi: 10.20944/preprints201907.0351.v1).

37. Shamshirband, S.; Hadipoor, M.; Baghban, A.; Mosavi, A.; Bukor J.; Annamaria R. Varkonyi-Koczy, Developing an ANFIS-PSO Model to predict mercury emissions in Combustion Flue Gases. Preprints 2019, 2019070165 (doi: 10.20944/preprints201907.0165.v1).

38. Shamshirband, S., et al., Ensemble models with uncertainty analysis for multi-day ahead forecasting of chlorophyll a concentration in coastal waters. Engineering Applications of Computational Fluid Mechanics, 2019. 13(1): p. 91-101.

39. Shamshirband, S., A. Mosavi, and T. Rabczuk, Particle swarm optimization model to predict scour depth around bridge pier. arXiv preprint arXiv:1906.08863, 2019.

40. Taherei Ghazvinei, P., et al., Sugarcane growth prediction based on meteorological parameters using extreme learning machine and 
artificial neural network. Engineering Applications of Computational Fluid Mechanics, 2018. 12(1): p. 738-749.

41. Torabi, M., et al., A Hybrid clustering and classification technique for forecasting short-term energy consumption. Environmental Progress and Sustainable Energy, 2019. 38(1): p. 66-76.

42. Torabi, M., et al., A Hybrid Machine Learning Approach for Daily Prediction of Solar Radiation, in Lecture Notes in Networks and Systems. 2019, Springer. p. 266-274.

43. Biswas, M., et al., State-of-the-art review on deep learning in medical imaging. Frontiers in Bioscience - Landmark, 2019. 24(3): p.392-426.

44. Bote-Curiel, L., et al., Deep learning and big data in healthcare: A double review for critical beginners. Applied Sciences (Switzerland), 2019. 9(11).

45. Feng, Y., H.S. Teh, and Y. Cai, Deep Learning for Chest Radiology: A Review. Current Radiology Reports, 2019. 7(8).

46. Griffiths, D. and J. Boehm, A Review on deep learning techniques for 3 Densed data classification. Remote Sensing, 2019. 11(12).

47. Gupta, A., et al., Deep Learning in Image Cytometry: A Review. Cytometry Part A, 2019. 95(4): p. 366-380.

48. Ha, V.K., et al., Deep Learning Based Single Image Super-resolution: A Survey. International Journal of Automation and Computing, 2019. 16(4): p. 413-426. 
49. Jiang, W., C.S. Zhang, and X.C. Yin, Deep Learning Based Scene Text Detection: A Survey. Tien Tzu Hsueh Pao/Acta Electronica Sinica, 2019. 47(5): p. 1152-1161.

50. Johnsirani Venkatesan, N., C. Nam, and D.R. Shin, Deep Learning Frameworks on Apache Spark: A Review. IETE Technical Review (Institution of Electronics and Telecommunication Engineers, India), 2019. 36(2): p. 164-177.

51. Li, X., Y. He, and X. Jing, A survey of deep learning-based human activity recognition in radar. Remote Sensing, 2019. 11(9).

52. Litjens, G., et al., State-of-the-Art Deep Learning in Cardiovascular Image Analysis. JACC: Cardiovascular Imaging, 2019. 12(8P1): p. 1549-1565.

53. Liu, S., et al., Deep Learning in Medical Ultrasound Analysis: A Review. Engineering, 2019. 5(2): p. 261-275.

54. Mazurowski, M.A., et al., Deep learning in radiology: An overview of the concepts and a survey of the state of the art with focus on MRI. Journal of Magnetic Resonance Imaging, 2019. 49(4): p. 939-954.

55. Narendra, G. and D. Sivakumar, Deep learning based hyperspectral image analysis-a survey. Journal of Computational and Theoretical Nanoscience, 2019. 16(4): p. 1528-1535.

56. Wang, H., et al., A review of deep learning for renewable energy forecasting. Energy Conversion and Management, 2019. 198. 
57. Wang, Y., et al., Enhancing transportation systems via deep learning: A survey. Transportation Research Part C: Emerging Technologies, 2019. 99: p. 144-163.

58. Zhang, W., et al., Deep learning-based multimedia analytics: A review. ACM Transactions on Multimedia Computing, Communications and Applications, 2019. 15(1s).

59. Zhou, J., et al., Deep learning for aspect-level sentiment classification: Survey, vision, and challenges. IEEE Access, 2019. 7: p. 78454-78483.

60. Zeiler, M.D. and R. Fergus. Visualizing and understanding convolutional networks. in European conference on computer vision. 2014. Springer.

61. Szegedy, C., et al. Going deeper with convolutions. in Proceedings of the IEEE conference on computer vision and pattern recognition. 2015.

62. Simonyan, K. and A. Zisserman, Very deep convolutional networks for large-scale image recognition. arXiv preprint arXiv:1409.1556, 2014.

63. Krizhevsky, A., I. Sutskever, and G.E. Hinton. Imagenet classification with deep convolutional neural networks. in Advances in neural information processing systems. 2012.

64. He, K., et al. Deep residual learning for image recognition. in Proceedings of the IEEE conference on computer vision and pattern recognition. 2016.

65. Kong, Z., et al., Condition monitoring of wind turbines based on spatiotemporal fusion of SCADA data by convolutional neural networks and gated recurrent units. Renewable Energy, 2020. 146: p. 760-768. 
66. Lossau, T., et al., Motion estimation and correction in cardiac CT angiography images using convolutional neural networks. Computerized Medical Imaging and Graphics, 2019. 76.

67. Bhatnagar, S., et al., Prediction of aerodynamic flow fields using convolutional neural networks. Computational Mechanics, 2019. 64(2): p. 525-545.

68. Nevavuori, P., N. Narra, and T. Lipping, Crop yield prediction with deep convolutional neural networks. Computers and Electronics in Agriculture, 2019. 163.

69. Ajami, A., et al., Identifying a slums' degree of deprivation from VHR images using convolutional neural networks. Remote Sensing, 2019. 11(11).

70. Min, S., B. Lee, and S. Yoon, Deep learning in bioinformatics. Briefings in bioinformatics, 2017. 18(5): p. 851-869.

71. Zhu, S., et al., Gaussian mixture model coupled recurrent neural networks for wind speed interval forecast. Energy Conversion and Management, 2019. 198.

72. Pan, B., X. Xu, and Z. Shi, Tropical cyclone intensity prediction based on recurrent neural networks. Electronics Letters, 2019. 55(7): p. 413415.

73. Bisharad, D. and R.H. Laskar, Music genre recognition using convolutional recurrent neural network architecture. Expert Systems, 2019. 
74. Zhong, C., et al., Inland Ship Trajectory Restoration by Recurrent Neural Network. Journal of Navigation, 2019.

75. Jarrah, M. and N. Salim, A recurrent neural network and a discrete wavelet transform to predict the Saudi stock price trends. International Journal of Advanced Computer Science and Applications, 2019. 10(4): p. $155-162$.

76. Al Rahhal, M.M., et al., Deep learning approach for active classification of electrocardiogram signals. Information Sciences, 2016. 345: p. 340-354.

77. Yin, Z. and J. Zhang, Cross-session classification of mental workload levels using EEG and an adaptive deep learning model. Biomedical Signal Processing and Control, 2017. 33: p. 30-47.

78. Sun, W., B. Zheng, and W. Qian, Automatic feature learning using multichannel ROI based on deep structured algorithms for computerized lung cancer diagnosis. Computers in biology and medicine, 2017. 89: p. 530-539.

79. Chen, Y., et al., Indoor location method of interference source based on deep learning of spectrum fingerprint features in Smart CyberPhysical systems. Eurasip Journal on Wireless Communications and Networking, 2019. 2019(1).

80. Liu, P., P. Zheng, and Z. Chen, Deep learning with stacked denoising auto-encoder for short-term electric load forecasting. Energies, 2019. 12(12). 
81. Nicolai, A. and G.A. Hollinger, Denoising Autoencoders for LaserBased Scan Registration. IEEE Robotics and Automation Letters, 2018. 3(4): p. 4391-4398.

82. Yue, L., et al., Multiple Auxiliary Information Based Deep Model for Collaborative Filtering. Journal of Computer Science and Technology, 2018. 33(4): p. 668-681.

83. Roy, S.S., M. Ahmed, and M.A.H. Akhand, Noisy image classification using hybrid deep learning methods. Journal of Information and Communication Technology, 2018. 17(2): p. 233-269.

84. Tan, Z., et al., Denoised Senone I-Vectors for Robust Speaker Verification. IEEE/ACM Transactions on Audio Speech and Language Processing, 2018. 26(4): p. 820-830.

85. Zhang, Q., et al., Deep learning based classification of breast tumors with shear-wave elastography. Ultrasonics, 2016. 72: p. 150-157.

86. Wulsin, D., et al., Modeling electroencephalography waveforms with semi-supervised deep belief nets: fast classification and anomaly measurement. Journal of neural engineering, 2011. 8(3): p. 036015.

87. Patterson, J. and A. Gibson, Deep Learning: A Practitioner's Approach. 2017: " O'Reilly Media, Inc.".

88. Vieira, S., W.H. Pinaya, and A. Mechelli, Using deep learning to investigate the neuroimaging correlates of psychiatric and neurological disorders: Methods and applications. Neuroscience \& Biobehavioral Reviews, 2017. 74: p. 58-75. 
89. Hassan, M.M., et al., Human emotion recognition using deep belief network architecture. Information Fusion, 2019. 51: p. 10-18.

90. Cheng, Y., et al., Deep belief network for meteorological time series prediction in the internet of things. IEEE Internet of Things Journal, 2019. 6(3): p. 4369-4376.

91. Yu, Y., et al., Forecasting a short-term wind speed using a deep belief network combined with a local predictor. IEEJ Transactions on Electrical and Electronic Engineering, 2019. 14(2): p. 238-244.

92. Zheng, J., X. Fu, and G. Zhang, Research on exchange rate forecasting based on deep belief network. Neural Computing and Applications, 2019. 31: p. 573-582.

93. Ahmad, M., et al., Deep Belief Network Modeling for Automatic Liver Segmentation. IEEE Access, 2019. 7: p. 20585-20595.

94. Ronoud, S. and S. Asadi, An evolutionary deep belief network extreme learning-based for breast cancer diagnosis. Soft Computing, 2019.

95. Ghimire, S., et al., Deep solar radiation forecasting with convolutional neural network and long short-term memory network algorithms. Applied Energy, 2019.

96. Hong, J., Z. Wang, and Y. Yao, Fault prognosis of battery system based on accurate voltage abnormity prognosis using long short-term memory neural networks. Applied Energy, 2019.

97. Krishan, M., et al., Air quality modelling using long short-term memory (LSTM) over NCT-Delhi, India. Air Quality, Atmosphere and Health, 2019. 12(8): p. 899-908. 
98. Zhang, R., et al., Deep long short-term memory networks for nonlinear structural seismic response prediction. Computers and Structures, 2019. 220: p. 55-68.

99. Hua, Y., et al., Deep Learning with Long Short-Term Memory for Time Series Prediction. IEEE Communications Magazine, 2019. 57(6): p. 114-119.

100. Zhang, J., et al., Short-term forecasting and uncertainty analysis of wind turbine power based on long short-term memory network and Gaussian mixture model. Applied Energy, 2019: p. 229-244.

101. Vardaan, K., et al., Earthquake trend prediction using long short-term memory RNN. International Journal of Electrical and Computer Engineering, 2019. 9(2): p. 1304-1312.

102. Mesri Gundoshmian, T., Ardabili, S., Mosavi, A., Varkonyi-Koczy, A., Prediction of combine harvester performance using hybrid machine learning modeling and re-sponse surface methodology, Preprints 2019.

103. Ardabili, S., Mosavi, A., Varkonyi-Koczy, A., Systematic review of deep learning and machine learning models in biofuels research, Preprints 2019.

104. Ardabili, S., Mosavi, A., Varkonyi-Koczy, A., Advances in machine learning model-ing reviewing hybrid and ensemble methods, Preprints 2019.

105. Ardabili, S., Mosavi, A., Varkonyi-Koczy, A., Building Energy information: demand and consumption prediction with Machine Learning models for sustainable and smart cities, Preprints 2019. 
106. Ardabili, S., Mosavi, A., Dehghani, M., Varkonyi-Koczy, A., Deep learning and machine learning in hydrological processes climate change and earth systems a systematic review, Preprints 2019.

107. Mohammadzadeh D., Karballaeezadeh, N., Mohemmi, M., Mosavi, A., Varkonyi-Koczy A., Urban Train Soil-Structure Interaction Modeling and Analysis, Preprints 2019.

108. Mosavi, A., Ardabili, S., Varkonyi-Koczy, A., List of deep learning models, Preprints 2019.

109. Nosratabadi, S., Mosavi, A., Keivani, R., Ardabili, S., Aram, F., State of the art survey of deep learning and machine learning models for smart cities and urban sustainability, Preprints 2019. 